\title{
VARIABLE FREQUENCY RFQS AS CYCLOTRON INJECTORS
}

\author{
A. Schempp \\ Institut für Angewandte Physik, J.W. Goethe-Universität, D-60054 Frankfurt, Germany
}

\begin{abstract}
Injectors for cyclotrons must provide low emittance beams with variable energy and bunch repetition frequency. The parameters of standard RFQs, which are short duty factor and constant operating frequency are not favourable for cyclotron application. Our development of the VE-RFQ has been adopted to match the HMIcyclotron at HMI in Berlin as a first variable frequency RFQ operating in cw-mode. Another example ist the RFQ at RIKEN which injects into the RILAC variable frequency linac. The layout and properties of these RFQs will be described.
\end{abstract}

\section{INTRODUCTION}

RFQ linacs use electrical $\mathrm{rf}$ focusing and are able to capture, bunch and accelerate high current ion beams at low energies e.g. directly from ion source extraction energies up to several $\mathrm{MeV}[1,2]$. Thus it can replace static preinjectors, which is especially favourable in combination with sources such as an ECR, because the source can be close to ground potential and is easy to operate and to service. The RFQ concept of spatially homogeneous strong focusing employs strong focusing with rf-electrical focusing which is independent of velocity and therefore the acceleration can start at a low energy with very short cells, because there are no limiting effects of lens ends and effective lengths like in static transport systems. This allows a large number of cells and by changing the stable phase of acceleration from $90^{\circ}(\mathrm{dc}$ beam) to the final value of e.g. $30^{\circ}$ in 100 small steps the dc-beam from the ion source is converted into a bunched beam. This adiabatic capture can have typically $90 \%$ transmission and a very small emittance growth.

Typical RFQ structures operate at 200- $400 \mathrm{MHz}$ for protons, at around $100 \mathrm{MHz}$ for heavy ions, and down to $20 \mathrm{MHz}$ for singly charged heavy ions, mostly at very low duty factors below 1\% [3]. Another typical RFQ-property is the fixed velocity profile given by the mechanical modulation of the electrodes, like the drift tube arrangement of DTLs, which requires a fixed input energy per nucleon and gives a fixed output energy per nucleon.

The mechanical modulation of the electrodes, as indicated in figure 1 , adds an accelerating axial field component to the homogeneous transport channel. Therefore the RFQ-structure accelerates and focuses with the same rf-fields.
For a given injection energy and frequency $\mathrm{f}$ the focusing gradient $\mathrm{G}=\mathrm{X} * \mathrm{U}_{\mathrm{Q}} / \mathrm{a}^{2}\left(\mathrm{U}_{\mathrm{Q}}=\right.$ electrode voltage, $\mathrm{a}=$ beam aperture, $\mathrm{X}<1$ for modulated electrodes) determines the acceptance in a low current application.

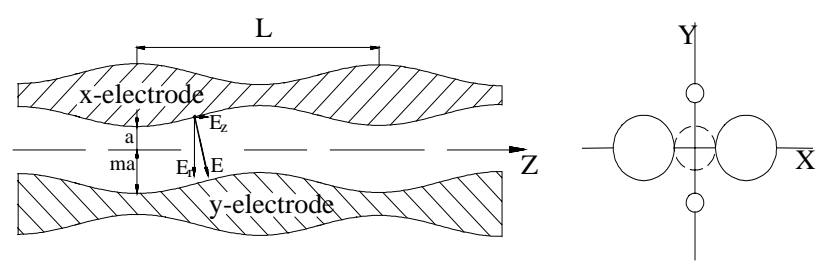

Fig. 1 Scheme of RFQ-electrodes

Generally, a maximum voltage $\mathrm{U}_{\mathrm{Q}}$ has to be applied at a minimum beam aperture a, because the radial focusing strength is the limiting factor. The highest possible operating frequency should be chosen to keep the structure short and compact. A lower frequency gives longer cells, stronger focusing and lower tolerance requirements. Optimisation with respect to specific applications may results in very different parameters. So a maximum transmission will result in a slow change of $\mathrm{m}$ and $\phi s$ and a long structure. Short (and cheap) structures will push or omit the adiabatic bunching process and reduce transmission and acceptance. The RFQ with highest effective accelerating voltage may have no adiabatic bunching section (a classical prebuncher instead) and will just accelerate, saving rf-power, length and costs.

\section{THE VE-RFQ STRUCTURE}

The fixed velocity profile is typical for RFQs. It can only be changed by varying the cell length L (by exchanging the electrodes) or the operation frequency $f$. The second possibility of changing the Wideröe [5] resonance condition: $\mathrm{L}=\beta_{\mathrm{p}} \lambda_{\mathrm{O}} / 2=\mathrm{v}_{\mathrm{p}} / 2 \mathrm{f}\left(\beta_{\mathrm{p}}=\mathrm{v}_{\mathrm{p}} / \mathrm{c}\right.$ particle velocity), is the way which has been used for RFQs with variable energy (VE-RFQ) [4]. For this reason it's possible to change the output energy using the same electrode system by varying the resonance frequency of the cavity: $\mathrm{vp} \sim \mathrm{f}, \mathrm{T} \sim \mathrm{v}_{\mathrm{p}}{ }^{2}$

To change the frequency of the 4-Rod-RFQ, a type of RFQ resonator developed in Frankfurt [5], the resonator can be tuned capacitively or inductively. Figure 2 shows the latter way of tuning by a movable tuning plate, which varies the effective length of the stems. 


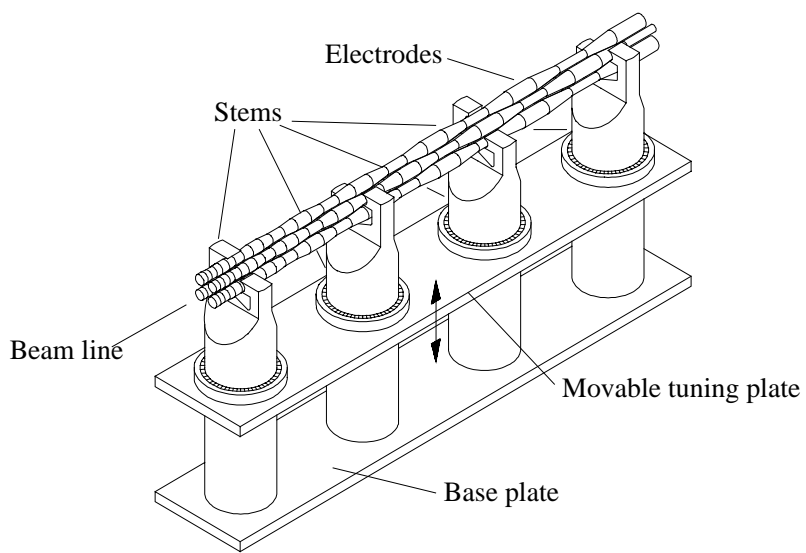

Fig. 2 Scheme of the VE-RFQ

In Frankfurt the VE-RFQ was developed for application as a cluster post accelerator at the IPNL in Lyon [6] and a first combination of an ECR ion source with an VE-RFQ for the IKF Frankfurt. Both operated in the FM range from 85 to $120 \mathrm{MHz}$, for which the structure could be built compact and rf-transmitters were available.

For application as injector for Separated SectorCyclotrons the frequency should be as low as $10-30 \mathrm{MHz}$ or a harmonic operation could be used.

Based on this development planning for a new injector for the ISL heavy ion cyclotron at the HMI Berlin (the former VICKSI machine) started in 92 . The ECR source together with two VE-RFQs should replace the 8UDTandem injector to match the new scientific program at the ISL which has been changed from nuclear physics to solid state physics $[7,8]$.

\section{THE ISL VE-RFQ}

The ISL is an isochronous cyclotron with four separated sectors. It has an external injection of beams with variable energy from either a CN-Van-de-Graaff or the new VE-RFQ. Figure 3 shows the layout of the accelerator complex.

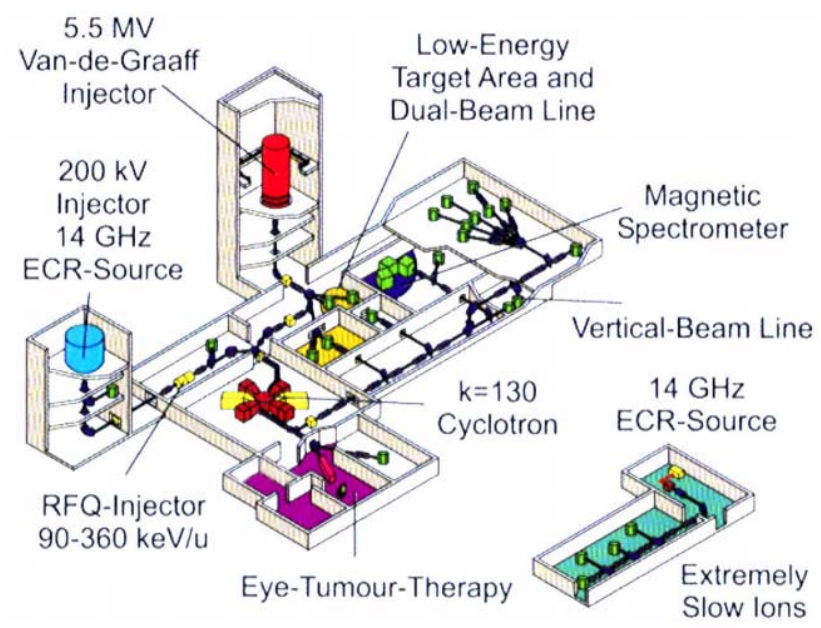

Fig. 3 Scheme of the heavy ion accelerator complex
To get higher intensities in the energy range between 2 to $6 \mathrm{MeV} / \mathrm{u}$, the former tandem injector has been replaced by a combination of an ECR ion source on a $200 \mathrm{kV}$ platform and a VE-RFQ as injector. This combination accelerates the ions to energies between $0.09 \mathrm{MeV} / \mathrm{u}$ and $0.36 \mathrm{MeV} / \mathrm{u}$ to cover the range of final energies out of the cyclotron between 1.5 and $6 \mathrm{MeV} / \mathrm{u}$.

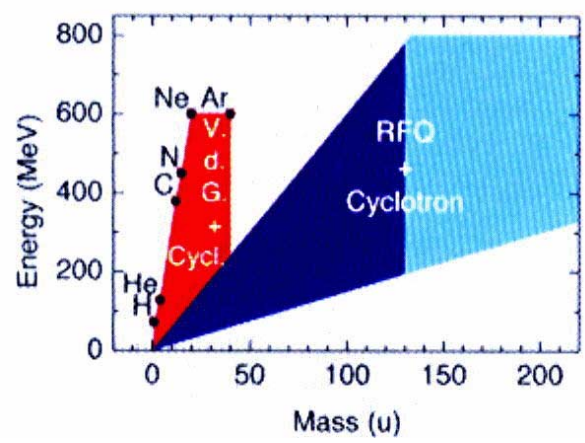

Fig. 4 Energy mass plot for the ISL-RFQ

Table 1: Parameters of the ISL-RFQ injector.

\begin{tabular}{|c|c|}
\hline HMI - RFQ: & \\
\hline min./max. E $_{\text {in }}$ & $15.16 / 29.72[\mathrm{keV} / \mathrm{u}]$ \\
\hline min./max. E $_{\text {out }}$ RFQ 1 & $90.98 / 178.35[\mathrm{keV} / \mathrm{u}]$ \\
\hline min./max. Eut $_{\text {Out }}$ RFQ & $178.35 / 355.09[\mathrm{keV} / \mathrm{u}]$ \\
\hline Energy gain factor RFQ 1/2 & $6 / 1.96$ \\
\hline Charge-to-mass-ratio & $>1 / 8$ \\
\hline Frequency & $85-120[\mathrm{MHz}]$ \\
\hline Electrode voltage $($ max. $)$ & $50[\mathrm{kV}]$ \\
\hline Length/diameter & $3 / 0.5[\mathrm{~m}]$ \\
\hline
\end{tabular}

Table 1 shows some characteristic parameters, figure 4 the mass - energy diagramm for the ISL. Several features are unique for this "Cyclotron injector RFQ": The operation has to be $\mathrm{cw}$, the frequency is variable and, to cover the large energy range, the RFQ is split in two sections.

There are two modes of operation: While the first section RFQ1 is assumed always accelerating, the second one RFQ2 can also accelerate or operate in the transport mode. There values for the phase and amplitude are chosen such that the bunched beam from RFQ1 is not captured in a bucket but just transported without acceleration in RFQ2. The matching between the two stages has to be done be proper choice of electrode parameters, amplitude of RFQ2 and relative rf-phase.

Fig. 5 shows the energy of the particles with highest and lowest energy in the bunch as function of the relative phase of the RFQ2. The arrow indicates a possible phase for the transport mode.

By this new method the energy could be changed by a factor 4 though the frequency is just covering the FMrange. This keeps the resonator compact and stable and the system cheap. 


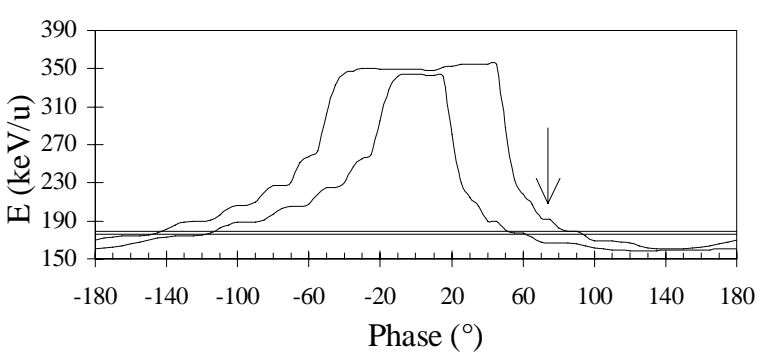

Fig. 5 Output energy band as functon of the relative phase between the two RFQs

The beam dynamics design has been optimised and the RFQ resonator has been designed resulting in a short structure with a total length of only $3 \mathrm{~m}$ and with an accelerating voltage of up to $2.8 \mathrm{MV}$. The RFQ has been built and the time consuming alignment and the tuning had been done at the NTG plant as well has first high power rf-test with the Bertronix Amplifiers (9). After transportation to HMI the RFQ has been set up at the ECR beam line and the new injector system has been tested $(10,11)$.

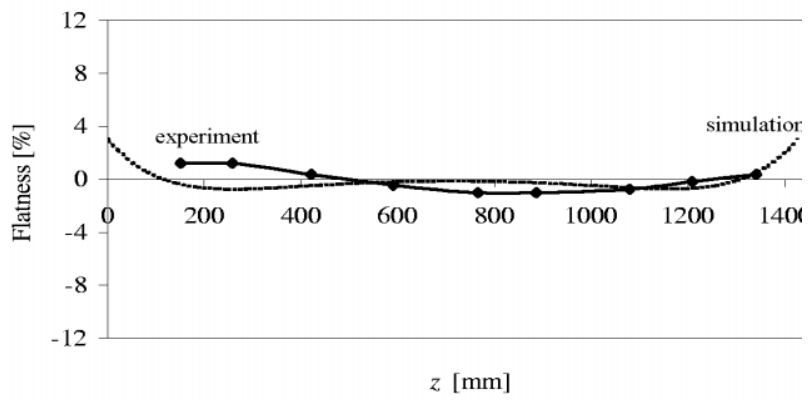

Fig 6 Field distribution in RFQ2 for the highest frequency

Fig 6 shows measured field distributions at the the highest frequency of $120 \mathrm{MHz}$, where the field variation is the highest, because the ratio of stem length to electrode length between stems is maximum. This corresponds to the radial voltage variation of a coaxial driven cyclotron resonator.

The measured frequency range of the RFQs is as designed. After some iterations the fast tuners could be shaped in a way that their range of up to $400 \mathrm{kHz}$ could balance the thermal detuning at a given rough tuner position without coming too close to resonance themselves. A special feature of the rf-coupling loop is that its mechanical position can stay fixed also with variable frequency. This speeds up the tuning.

The beam tests showed that the results come close to the beam dynamics simulations. Fig. 7 and figure 8 show comparisons for the phase variation of RFQ2, which can be seen as changing the polarities and amplitudes in the quadrupole chain to which the second RFQ can be treated if not properly phased for acceleration and transmission . Fig. 9 an energy spectrum of a $\mathrm{O}^{4+}$ beam in high energy mode.

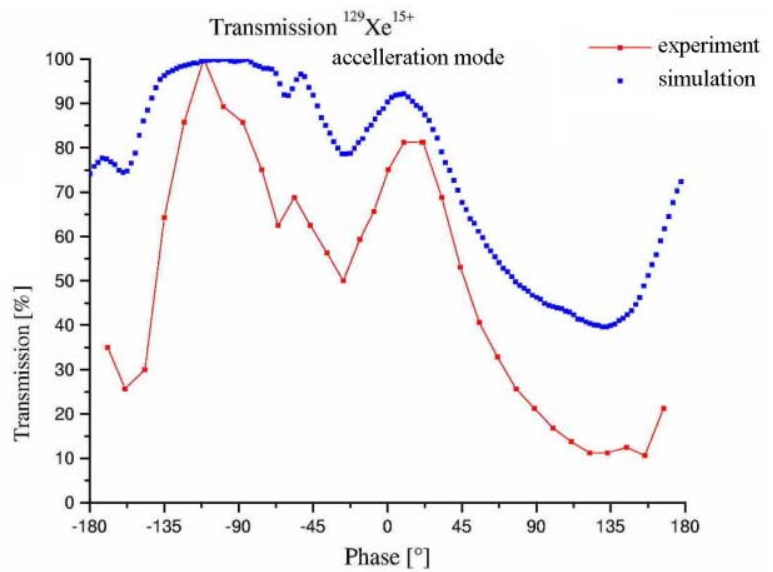

Fig. 7 Transmission in accelering mode as function of the phase between the RFQs

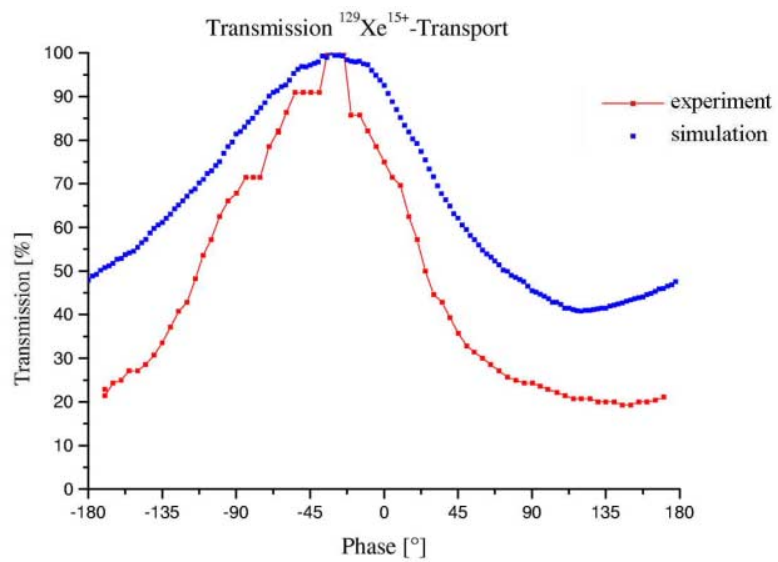

Fig. 8 Transmission in transport mode as function of the phase between the RFQs

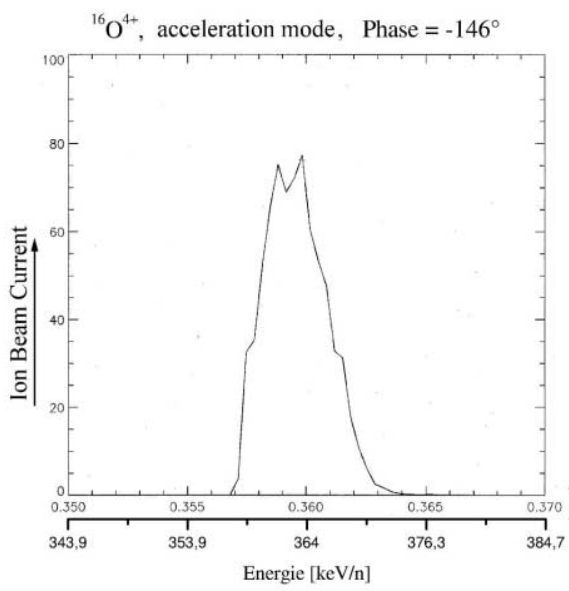

Fig. 9 Energy spectrum at full energy (RFQ1, RFQ2 accelerating) 


\section{RIKEN - RFQ}

For heavy ions a frequency tunable Linac (RILAC)[12] serves as injector for the $\mathrm{K}-540 \mathrm{MeV}$ ring cyclotron (RRC). The RILAC has two preinjectors, a CockroftWalton dc-preinjector (CW) and the new VE-RFQ [13]. The injector layout is shown in figure 10 .
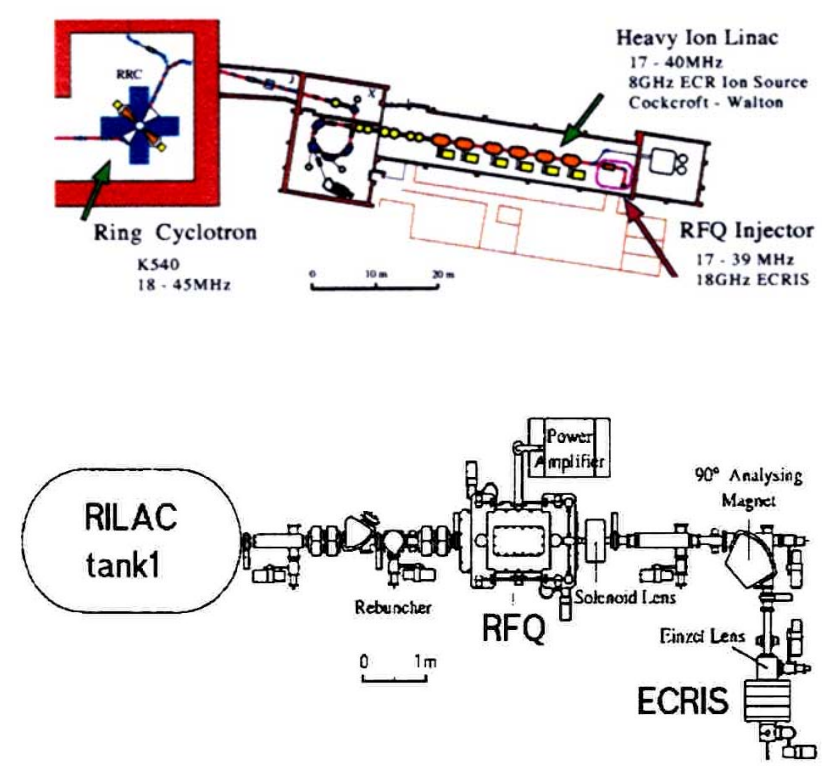

Fig 10 Layout of the RFQ-Injector at RIKEN

For a maximum beam transmission from the ECR source to the target the RFQ has to match the beam time structure RILAC and RRC. The most difficult problem to be solved is the large frequency tuning of $17-40 \mathrm{MHz}$. For the heaviest particles at the highest RILAC frequency the RFQ-preinjector has to supply an equivalent maximum voltage of $450 \mathrm{kV}$.

Using the same $\lambda / 4$ resonator concepts like RILAC tanks, a compact low frequency RFQ-structure has been developed. This folded coaxial structure (FCRFQ) is an asymmetric structure, the beam axis is not on ground potential resp. one electrode pair is on ground. This creates some beam matching problems at low energies but allows to tune the frequency range with a massive single radial $\lambda / 4$-line as shown in figure 11 .

The resonator is loaded by the electrode capacity (appr. $250 \mathrm{pF} / \mathrm{m}$ ). Therefore the length of the radial stub line is only $80 \mathrm{~cm}$ for the lowest frequency. To ensure mechanical stability, the electrode line is supported by ceramic pillars.

The critical point in these VE-RFQ structures is the operation at the highest frequency, where the power consumption, the losses in the ceramics are at maximum and the ratio of lengths of the stub line to the electrodes is small. The maximum vane voltage in the RIKEN-RFQ is $36.8 \mathrm{kV}$. The required rf-power of $26 \mathrm{~kW}$ is fed in capacitively, close to the electrodes.

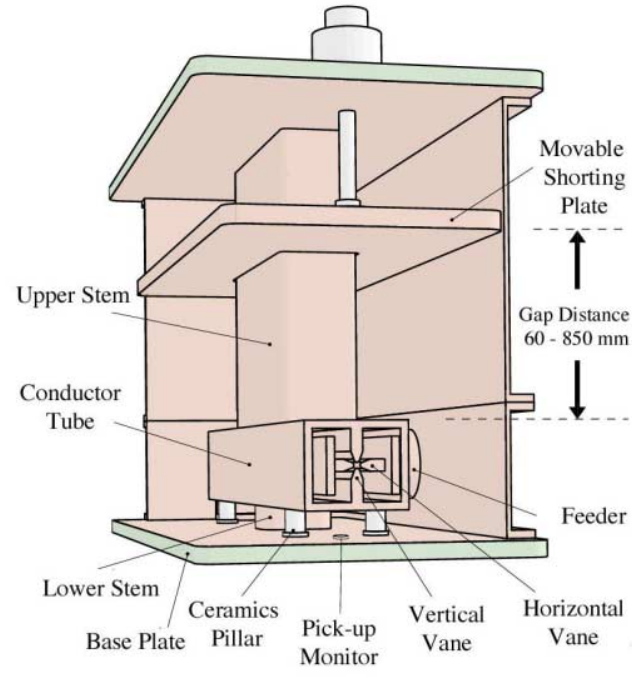

Fig. 11 Scheme of the FCRFQ

The new beam dynamics design results in RFQelectrodes with a length of $1.53 \mathrm{~m}$, with high acceptance and good transmission. The parameters of the RIKENRFQ are summarized in table I. The RFQ has been built and is now operating routinely. Fig. 12 shows the variety of beams which have been produced.

Table 2 Parameters of the RILAC-RFQ injector

\begin{tabular}{|l|c|}
\hline max. $E_{\text {in }}$ & $20\left[\mathrm{keV}^{*} \mathrm{q}\right]$ \\
\hline min./max. E $_{\text {Out }}$ RFQ 1 & $18 / 90[\mathrm{keV} / \mathrm{u}]$ \\
\hline Energy gain factor & 22.5 \\
\hline Stroke of tuner & $79 \mathrm{~cm}$ \\
\hline mass- to - charge - ratio & $6-26$ \\
\hline Frequency & $17.4-39[\mathrm{MHz}]$ \\
\hline Electrode voltage (max.) & $36.8[\mathrm{kV}]$ \\
\hline Cavity Length/diameter & $1.7 / 1 \mathrm{x} 0.7[\mathrm{~m}]$ \\
\hline
\end{tabular}

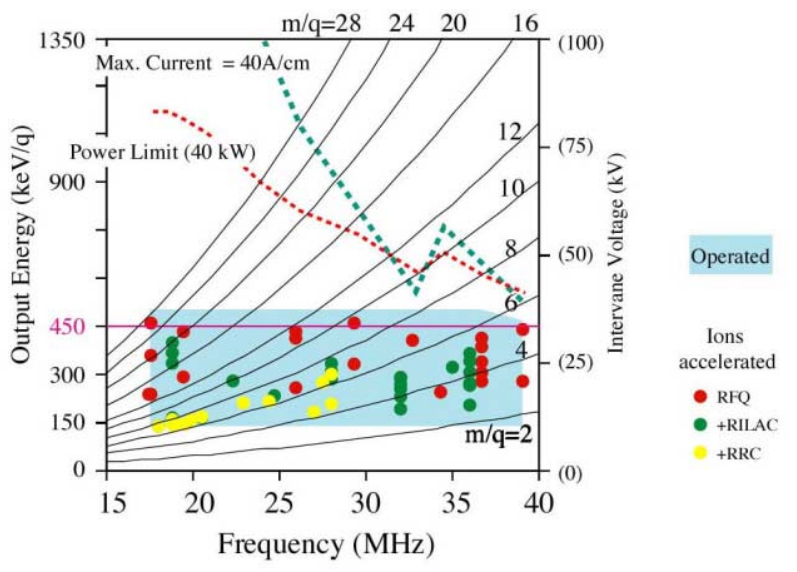

Fig. 12 Summary of beam experiment at the FCRFQ 


\section{CONCLUSION}

These two successful projects demonstrate that RFQs can be used as cyclotron injectors. Especially the combination with

ECR sources have favourable properties. Phase width and energy resolution are sufficient to give minimum losses in the cyclotron. Overall transmissions (source to target) of over $30 \%$ have been measured.

\section{ACKNOWLEDGEMENTS}

The author is grateful to O. Kamigaito and A. Goto for the information on the RIKEN RFQ.

He wants to thank H. Homeyer and W. Pelzer for the collaboration on the RFQ-project.

During that time two Diploma- and one $\mathrm{PhD}$ thesis were done on, with or about the HMI VE-RFQ project (F. Marhauser, F. Höllering, O. Engels). They converted sketches, ideas, thoughts, estimates into the real thing. They put in a lot of effort and own ideas. They are the ones to be blamed for any deficiencies of that injector system. It was a pleasure to work with them.

Thanks to I. Müller, whose mastery of electronics was crucial for making things work.

After successful completion a special thank to $\mathrm{H}$. Homeyer for taking the risk of such an ambitious project.

This work was supported by the BMBF

\section{REFERENCES}

[1] I.M. Kapchinskij and V. Teplyakov, Prib. Tekh. Eksp 119, No.2 (1970) 17

[2] K.R. Crandall, R.H. Stokes, T.P. Wangler, Linac 79, BNL 51134 (1979) 205 A. Schempp, Adv. of Acc. Physics and Techn.12, World Scientific (1993) 194

[4] A. Schempp, NIM B40/41 (1989) 937

[5] A. Schempp, Habilitationsschrift, Universität Frankfurt am Main (1990)

[6] M.J. Gaillard et al., Z.Phys. D26, (1993) 347

[7] H. Homeyer. W. Pelzer, Vorschlag zur Realisierung eines Ionenstrahllabors im HMI (ISL-Berlin), HMIBerlin, 1992

[8] B. Martin et al., Proc. $14^{\text {th }}$ Cycl. Conf.1995, p. 78

[9] Neue Technologien, D-63571 Gelnhausen

[10] O. Engels, Dissertation, Univ. Frankfurt 1998

[11]F. Höllering, Diploma thesis, Univ. Frankfurt 1999

[12] M. Odera, LINAC 84, GSI 84-11 (1984) 36

[13] O. Kamigaito et al., Jpn. J. Appl. Phys. 34(1994)537

O. Kamigaito et al., RSI 70 (1999) 4523 